\title{
Redshift evolution of Tully-Fisher relation
}

\author{
Ismael Ferrero and Mario G. Abadi
}

Instituto de Astronomia Teorica y Experimental (IATE), Observatorio Astronomico de Cordoba (OAC) and CONICET, Laprida 922 X5000BGR Cordoba, Argentina

email: iferrero@oac.unc.edu.ar

\begin{abstract}
Using the EAGLE cosmological simulation of galaxy formation we test the ability of the $\Lambda \mathrm{CDM}$ cosmological model to reproduce the Tully-Fisher relation (TFR) and its redshift evolution. We find that our simulated galaxies follow a TFR that is in good agreement with observed results up to $z=1$, indicating no evolution in the slope and a weak decrease in the zero-point.
\end{abstract}

Keywords. galaxy:formation, galaxy: kinematics and dynamics, galaxy: structure

We use the EAGLE cosmological hydrodynamical simulation to study the redshif evolution of the Tully-Fisher relation (Tully \& Fisher 1977). We select simulated galaxies resembling observed spiral galaxies. Imposing at redshift $z=0$, a minimum stellar mass of $M_{*}>10^{9} \mathrm{M}_{\odot}$ and a ratio between rotational-to-total kinetic energy parameter $\kappa_{\text {rot }}=\Sigma V_{\text {rot }}^{2} / \Sigma V^{2}>0.6$ to ensure that our simulated galaxies are rotationally supported. Furthermore, we restrict our analysis to galaxies with half stellar mass radius $r_{\text {half }}$ at least three times larger that the softening length $\epsilon=0.7 \mathrm{kpc}$ to ensure that our objects are spatially well-resolved.

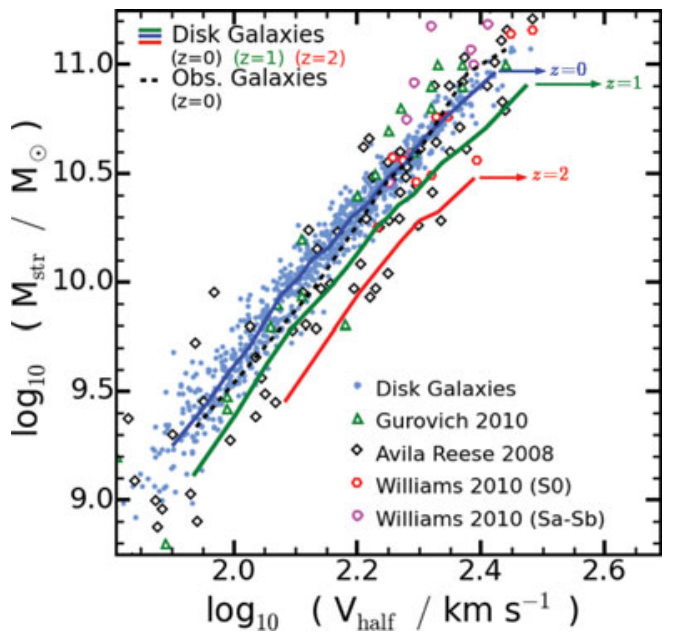
In the Figure we show the comparison of the TFR for simulated galaxies and observational data at redshift $z=0$ (see labels). Solid lines are medians for simulated galaxies for $z=0,1,2$ from top to bottom, respectively, and dashed line is median of observed data. A very good agreement can be seen between simulated and observed data. We found that at redshift $z=0$ our simulated disk galaxies have a TFR slope of 3.3 which keeps approximately constant with redshift but shows a systematic decrease by a factor 3 in its zero point from $z=0$ to redshift $z=2$. The simulated TFR slope is steep, $M_{\text {str }} \propto V_{\text {half }}^{3.3}$, but close to the virial theoretical expectation $\left(M_{200} \propto V_{200}^{3}\right)$. This higher slope can be trace back to the lower efficiency of smaller haloes to collect its baryonic material consistent with abundance matching predictions (see also Sales et al. 2016). The zero-point evolution interpreted as an evolution in the halo mass since changes in the stellar mass fraction seems to be counter-balanced by changes in the circular velocity profiles having no net effect.

\section{References}

Sales, L. V., Navarro, J. F., Oman, K., et al. 2016, arXiv:1602.02155

Tully, R. B. \& Fisher, J. R. 1977, A\&A, 54, 661 